\title{
Microprocessor device to monitor the level of emotional stress in humans
}

\begin{abstract}
Modern medical, biological and psychophysiological studies convincingly show emotional stress is the cause of many psychosomatic disorders, exerts a comprehensive destructive influence on the vital activity of an organism, undermines the health of people, reduces the reliability of human activity. Therefore, it is extremely important to control the degree of emotional stress development (its autonomic features) and to determine that dangerous border beyond which one can not go out. However, with all the significance and widespread prevalence of stress among the population, there are still no methods of measuring stress in people in real everyday life; full-fledged objective criteria of stress are not developed. The aim of the research is to develop an information system for everyday, objective self-control of stress level, with the help of which it would be possible to preserve health of stressed people.

On the basis of our experimental studies, an objective criterion of emotional stress, based on the calculation of cross-correlation coefficients between vital autonomic parameters: heart rate, respiratory rate and blood pressure, is proposed. It was found emotional stress is characterized by a decrease in cross-correlation between autonomic parameters, a disorder of biorhythms, due to the disintegration of the activity of functional systems. The methodology and information system for an objective assessment of emotional stress, which can be recommended for the building up of a specialized microprocessor system designed for comprehensive, rapid assessment of emotional stress level in sleep and wakefulness and prevention of disorders of vital functions, is developed.
\end{abstract}

Keywords: brain, psyche, consciousness, emotions, stress, respiratory apnea, autonomic indicators, cross-correlation analysis, microprocessor system
Volume 2 Issue 4 - 2017

\author{
Glazachev OS, 'Yumatov EA, ,',2,3 Grechikhin \\ $\mathrm{VA},{ }^{3}$ Kramm $\mathrm{MN},{ }^{3}$ Strelkov $\mathrm{NO}^{3}$ \\ 'IM Sechenov First Moscow State Medical University, Russia \\ 2PK Anokhin Institute of Normal Physiology, Russia \\ ${ }^{3}$ National Research University Moscow Power Engineering \\ Institute, Russia
}

Correspondence: Glazachev OS, IM Sechenov First Moscow State Medical University, Russia; Email glazachev@mail.ru

Received: April 27, 2017 | Published: May II, 2017

\section{Introduction}

Many people experience great emotional stress in everyday life. Without exaggeration, we can say that each person risks at any time to exceed his or her maximum permissible level of emotional stress, which can pose a danger to life. Emotional stress as a psycho-emotional state, develops in a person as a result of continuous negative emotions arising in conflictual behavioral situations with the impossibility or long-lasting difficulty in satisfying vital social or biological needs. ${ }^{1,2}$ Modern medical-biological and psychophysiological studies convincingly show emotional stress and its autonomic-humoral accompaniment are the cause of many psychosomatic diseases; it exerts a comprehensive destructive influence on the vital activity of the organism, undermines the health of people, reduces the work capacity of a person. The solution of the stress problem is primarily related to the need to control the degree of its development (autonomic-visceral representations) and to determine the dangerous border beyond which one cannot go out. ${ }^{3,4}$ However, for all the significance and widespread prevalence of stress among the population, there is still no appliances or devices to measure the level of stress in people in real everyday life. The purpose of this work is to develop a methodology for the information microprocessor system for everyday, objective selfmonitoring of the level of a person's psychoemotional state.

\section{Criteria for monitoring emotional stress}

Emotional tension is a systemic integration, practically involving all the visceral organs, processes and bodily functional systems. It has been established in emotional stress modelling experiment and during actual exam tension subjects begin to show the signs of autonomic functions disintegration. Core indicators of such autonomic disregulation can be revealed by cross-correlational analysis of blood pressure (BP), heart rate (HR) and breathing rate $(\mathrm{BR})$ variables as reliable decline of the "power" indicator for correlations in dynamically monitored autonomic parameters. ${ }^{5.6}$ Emotional stress affects and causes various sleep disorders. ${ }^{5-9}$ During sleep, as well as in wakefulness, the presence of reliable correlations between changes in heart rate and $\mathrm{BH}$ was revealed. Ben-Tal A et al. ${ }^{10}$ presents an overview of currently existing mathematical and computer models describing cardio-respiratory relationships and their changes in the transition from wakefulness to sleep. ${ }^{10}$ During sleep, there is a syndrome of respiratory apnea, which is characterized by a temporary stop of breathing, and can cause sudden death. Syndrome of respiratory apnea can be of central origin and its cause can be emotional stress. Harrington et al. ${ }^{11}$ showed the presence of reliable correlations between the heart rate variation (HRV) and BR in patients with a syndrome of respiratory apnea. ${ }^{11}$

The respiratory center provides rhythmic breathing. During wakefulness, the respiratory center is under the influence of the activating structures of the brain. In the sleep, the activity of the respiratory center is inhibited, and apnea occurs as a result of a decrease in the excitability of the respiratory neurons, and the sensitivity of the chemoreceptors to hypoxia and hypercapnia which leads to respiratory failure. The respiratory center is more sensitive to "drowsy" inhibitory influences than cardiac activity regulated by an 
autonomic nervous system. Therefore, in a dream, there can be more decrease in the correlation relationship between cardiac and respiratory rhythm than during wakefulness, which is especially pronounced in emotional overstrain. It is important that the microprocessor performs cross-correlation analysis of the cardiac and respiratory rhythm not only during wakefulness, but also in a dream. Studies have shown that emotional stress is characterized by a decrease in cross-correlation between these parameters, due to the disintegration of the activity of various functional systems of the organism, in which their normal relationships which determine the coordinated, multiply connected regulation of the vital signs of the organism are violated. A significant decrease in the "power" of the correlation indicates a dissociated change in individual parameters, a decrease in regulatory influences that ensure the consistency of visceral reactions. Emotional stress of a person is a systemic multi-level reaction of the body. This explains the fact that the criterion of emotional stress can serve as an integral indicator, collectively characterizing the systemic organization of the mental and neurophysiological activity of a human brain.

\section{Cross-correlation analysis of cardiac and respiratory rhythms}

Based on the conducted studies, we developed a priority method for individual objective quantitative assessment of the degree of development of emotional stress in order to prevent stress caused by functional and pathological disorders. ${ }^{3}$ The method is based on the registration of vegetative indices of the person: heart rate, $\mathrm{BH}$ and on the calculation of cross-correlation coefficients, which, as our studies have shown, objectively reflect the degree of development of emotional stress. ${ }^{12,13}$ The information system for analyzing the rhythms of the heart and respiration is built on the basis of a PC which makes it possible to modify and improve algorithms for processing incoming physiological information, visualizing and archiving the received data. For the analysis of the heart rhythm, any electrocardiographic lead can be selected, which provides the possibility of changing the position of the extremities in the measurement.

As a sensor for the analysis of the breathing rhythm, a miniature semiconductor thermal diode is used, which is easy to install with a clip in the nasal canal and in which, when a stable micro current is passed in the forward direction, the contact difference of potentials changes almost linearly with a temperature change. The interconnection unit of the sensors of physiological processes with the computer contains the cardiac signal channel and the respiratory signal channel. To register R-waves, the cardiac channel passes the frequency band $25 \mathrm{~Hz}-35 \mathrm{~Hz}$. In addition, the cardio channel features circuit-based measures for suppressing in-phase interference, typical for schemes of measuring the potential difference, and used in cardio amplifiers. ${ }^{14,15}$ In the developed coupling block for the suppression of in-phase noise, separate amplifiers of the potentials taken from the electrodes with a large input resistance (of the order of 3-5M $\Omega$ ) are used. These amplifiers are connected to a subtractive amplifier with precision resistors. The inspiratory and expiratory impulses are formed in the respiratory channel; they will determine the intervals of respiration in the program. A shimmer-type shaper, as well as a differentiating chain, is used to obtain these pulses. The interface unit is implemented on the basis of inexpensive microcircuits and components.

A sound card, which is part of almost all personal computers, is used to convert analog signals from the output of the conjugation unit to digital form. In particular, it is possible to program the sampling speed (sampling rate), and the maximum sampling frequency $(44.1 \mathrm{kHz})$ exceeds the possible upper frequencies in the spectra of the channel signals representing the physiological processes under study. We used the standard Win32 API Waveform Audio Interface, which allows converting the analog input signal to digital signal format with a specified sample rate and bit width. ${ }^{15}$ To ensure the continuity of the process of measurement and processing in real time, the digitization program provides the formation of a sequence of input buffers on each of the recording channels forming a conveyor, allowing simultaneous digitization of the signal and processing of digitized values. The program allows to set the following parameters of the digitization process: the sampling frequency, the number of input buffers, the number of samples in the buffer. The default values are:
i. Bit depth-16
ii. Sampling frequency, $\mathrm{Hz}-1000$
iii. Number of buffers-10
iv. Buffer size (counts)-500

The sampling rate is selected in such a way as to reliably fix the R-waves of the cardio signal and the moments of the inhalation/ exhalation pulses of the respiratory channel. To suppress interference from the circuits of industrial frequency, it is advisable to sample the input signals at a frequency that is a multiple of $50 \mathrm{~Hz}$. Preprocessing of digitized signals is performed using filters with finite impulse response (FIR filters). Heart and respiratory rhythms are characterized by interval sequences - sequences of time intervals between R-waves (RR-intervals) in the cardiac signal recording and between the impulses of the breath sensor signal. The tracking threshold method is used to record the moment of appearance of the current pulse. So, when searching current R-wave (or QRS-complex in cardiology), the reference thresholds in our algorithm

$$
U_{i}\left(t_{j}\right)=U_{0} \cdot e^{-\beta t}
$$

Where $U_{0}$-the level of the vertex of the last found $R$-wave; $U_{n}$ the threshold level at the current time $t_{j}=j / f_{D}$ (counted relative to the vertex of the last found $R$-wave); $f_{D}$ - sampling frequency; $\beta$-coefficient of attenuation of the threshold. The detection procedure here is the fact that the cardiac signal exceeds the threshold level. The moment of detection $i$-th $\mathrm{R}$-wave Thi, counted relative to the beginning of the cardiac signal recording, is used to determine the next cardio interval (RR-interval) $H_{i}=T h_{i}-T h_{i-1}$. Such an estimate of the cardiointerval is more accurate than the estimate of the interval between the vertexes of R-waves, since on the steep section the moment of crossing the threshold is recorded with a smaller error. After detecting the current pulse, the system finds its vertex and records the level $U_{0}$ and the time moment for this vertex. Then the search for the next R-wave begins. The coefficient of attenuation of the threshold is determined by the formula

$$
\beta=-\ln \frac{1}{\Delta U_{i}},
$$

Where $\Delta \mathrm{U}_{n}$ - relative decrease in the threshold level on an interval of 1 second.

In order not to re-discover the same R-wave in the case of an irregular ("cut") vertex of R-wave, an interval of insensitivity is 
formed, which begins after registration of the vertex and lasts for 50ms. During this time, physiologically, a new R-wave cannot appear, and at the same time the possibility of re-detection of the already considered complex disappears.

Thus, the operation of the algorithm can be divided into 3 stages:

i. Searching for the R-wave, the program compares the value of the threshold and the signal;

ii. Searching for the vertex of the R-wave and the determination of the initial threshold level $U_{0}$;

iii. Shift to the dead band interval of 50ms (in this interval the program does not form a threshold and does not detect any new R-wave).

The advantage of this method is the automatic adjustment of the threshold level to the real amplitude of the R-waves in the cardiac signal of this person. Because of the unpredictable spread in the amplitude of R-waves in different people, you can not use a fixed detection threshold. For example, if the level of the threshold is obviously too low, false triggers start because of interference in the recording. The algorithm for measuring the rhythm of breathing is analogous to the algorithm for measuring the rhythm of the heart. On the basis of recorded information, interval sequences are formed - the sequences of cardiointervals $H_{i}$ and respiratory intervals $B_{k}$ (here lower indexes mean current number of interval in record). Plots $H_{i}(i)$ and $B_{k}(k)$ will be called a cardiorhythmogram and pneumothymogram, respectively (Figure 1). It is impossible to determine the correlation coefficient directly between the sequences of cardiointervals $H_{i}$ and respiratory intervals $B_{k}$, since these intervals are recorded at different time points. At any observation interval, the number of cardiointervals recorded is, on average, 3-4 times greater than the number of respiratory intervals.

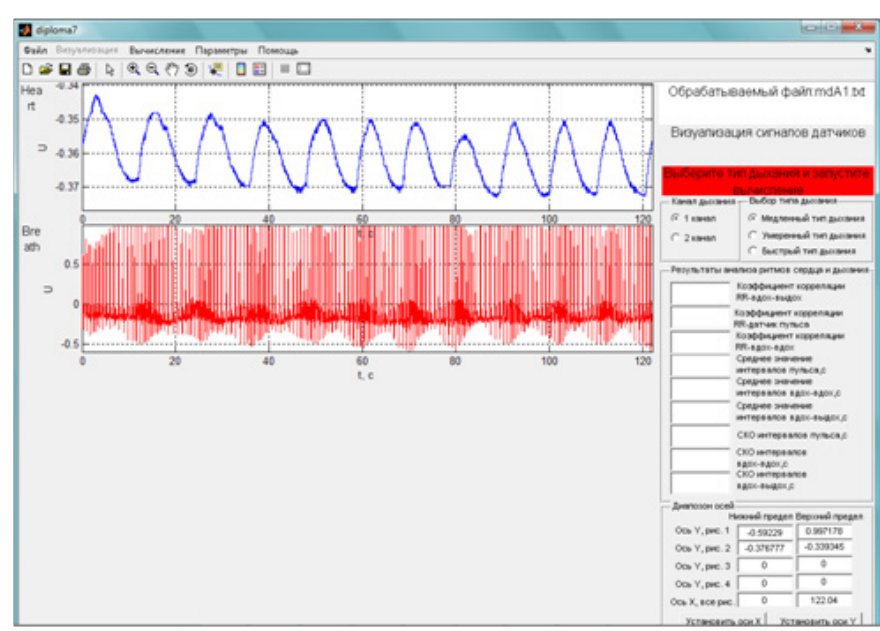

Figure I Correlation analysis of heart rate (HR) and breathing rate (BR).

Therefore, a method is used for the formation of interval sequences, taking into account the structure of the respiration process. In this case, we selected: pulses of positive polarity with a sign $B p=1$, corresponding to the beginning of the inspiratory phase, pulses of negative polarity (sign $B p=-1$ )- the beginning of exhalation, $T b_{k}^{-}$ time of $k$-th registration of the next pulse (irrespective of polarity); $V_{k}$-interval between adjacent breaths $(B p=1)$ or exhalations $(B p=-1)$; $B t_{k}$-exhaling-inhaling intervals $(B p=1)$ or inhaling- exhaling $(B p=-$ 1). Note that for brevity, here and elsewhere the words inhale and exhale signify the beginning of the phase of inhalation and exhalation, respectively.
Thus, the sequences $B t_{k}, B p_{k}$ and $V_{k}$ are defined at the time of registration of inhalation and exhalation $T b_{k}$. As for the cardiointervals $H_{i}$, as it was noted earlier, they are defined at the time of registration of $\mathrm{R}$-waves $T h_{i}$. It is convenient to interpolate the interval sequences by the moments of cardiac excitations $T h_{i}$ for the formation of modified sequences, determined at the same time points. The values of the respiratory intervals $B t_{k}, B p_{k}$ and $V_{k}$ we assigne to all points $T h_{i}$, relating to the respiratory interval $\left(T b_{k-1}, T b_{k}\right)$ :

$$
\begin{aligned}
& \tilde{B} t_{i}=B t_{k}, \tilde{B} p_{i}=B p_{k}, \text { if } T b_{k-1}<T h_{i}<T b_{k .} \\
& \text { Analogous } \\
& \tilde{V}_{i}=V_{k} \text {, if }\left(T b_{k-2}<T h_{i}<T b_{k}\right) \cap\left(B p_{k-2}=B p_{k}=+1\right),
\end{aligned}
$$

Taking into account that the inhaling- inhaling intervals correspond to the impulses of a positive-polarity respiratory sensor. The beginning of the formation of modified interval sequences must correspond to the first cardiointerval that satisfies the condition $T h_{i}>T b_{l}$. Similarly, the formation must be completed when $T h_{i}>T b_{N}$, where $N$ is the number of the last in the breath pulse record. Using modified interval sequences, one can, by analogy with (1), find estimates of the correlation coefficients between $\tilde{B} t_{i}, \tilde{B} p_{i}, \tilde{V}_{i}$ and $H_{i}: \widehat{r}(H, \tilde{B} t)$, $\widehat{r}(H, \tilde{B} p)$ and $\widehat{r}(H, \tilde{V})$.

The evaluation of the correlation coefficient between the obtained modified interval sequences is estimated according to the known formula:

$$
\widehat{r}(H, B)=\frac{\sum_{j=1}^{N}\left(B_{j}-\bar{B}\right) \cdot\left(H_{j}-\bar{H}\right)}{\sqrt{\sum_{j=1}^{N}\left(B_{j}-\bar{B}\right)^{2} \cdot \sum_{i=1}^{N}\left(H_{j}-\bar{H}\right)^{2}}},
$$

where $N=T_{A H} / \Delta t$ is total number of averaging intervals for the analysis time $T_{A H} ; \bar{H}, \bar{B}$ are average values of intervals during the study session. In this case, the confidence interval, in which with probability $D=1-\alpha$ the exact value of the correlation coefficient can be found, is defined as follows. ${ }^{15}$

$$
\operatorname{th}\left[\arg \operatorname{th}(r)-\frac{u_{\alpha}}{\sqrt{N-3}}\right]<r<\operatorname{th}\left[\arg \operatorname{th}(r)+\frac{u_{\alpha}}{\sqrt{N-3}}\right],
$$

Where the quantile $u_{\alpha}$ is determined from equation $\hat{O}\left(u_{\alpha}\right)=1-0.5 \cdot \alpha ; \quad \hat{O}(u)^{-}$-distribution function of a random variable with a standard normal distribution.

Along with cross-correlation analysis of cardiac and respiratory rhythms, the microprocessor system can monitor rhythmic breathing during night sleep. The program tracks the duration of a pause between consecutive breaths in each breathing cycle, and compares it with a fixed maximum allowable duration of the respiratory pause. If a prolonged respiratory arrest occurs, the microprocessor system produces a signal that activates the activity of the brain, in the form of an awakening sound or sensory stimulation. This prevents lifethreatening respiratory apnea. For the analysis of the psychoemotional state of a person, we developed information software and an experimental model that allows us to identify the minimum time for a person to perceive the visual image. ${ }^{16,17}$ With emotional stress, attention is "scattered" and the ability to comprehend information is reduced, which can be used as an additional test for everyday monitoring and recognition of a person's mental tension. 


\section{Conclusion}

Emotional stress exerts a comprehensive influence on the vital activity of the organism and undermines the health of people. To prevent disorders of physiological functions, it is necessary to control vegetative manifestations of emotional stress during wakefulness and sleep. On the basis of our experimental studies, objective criteria have been proposed and an information system for monitoring level of emotional stress. The algorithm of the information system has been worked out; specialized software based on cross-correlation analysis of cardio- and respiratory rhythms has been created. The developed methodology can be recommended for the creation of specialized, microprocessor-based equipment designed for comprehensive, rapid assessment of the level of emotional stress of a person during wakefulness and sleep and prevention of disorders of vital functions.

\section{Acknowledgements}

None.

\section{Conflict of interest}

The author declares no conflict of interest.

\section{References}

1. Sudakov KV, Yumatov EA. Emotional stress in contemporary life. 1991. $83 \mathrm{p}$.

2. Yumatov EA. Emotional stress: theoretical and clinical aspects. In: Sudakov KV, et al. editors. Committee on Press and Information. Russia: Springer; 1997. $168 \mathrm{p}$.

3. Yumatov EA. Information medical science-protection of people's life and health in everyday conditions. Biomedical Radioelectronics Journal. 2016;5:72-79.

4. Spielberger CD. Measuring stress in the workplace: health and police implications. Stress at the workplace: health and productive. Netherlands: Springer; 1995. $11 \mathrm{p}$.

5. Vein AM, Sudakov KV, Levin Ya I, et al. Stages of sleep after psychoemotional effects: individuality of changes. Physiological Journal. 2001;87(3):289-295.
6. Romanov AI, Reshetnyak VK. Sleep and its disorders. Russia: Slovo Publishing; 2003. 272 p.

7. Yumatov EA, Glazachev OS, Bykova EV, et al. Interrelation of emotional stress and sleep. Herald of the International Academy of Sciences. 2016;1:5-14.

8. Yumatov EA, Pertsov SS. System organization of the relationship of emotional stress and sleep. Journal of Neurology \& Neuroscience. 2016;7(5):145.

9. Yumatov EA. System organization of mental activity of the brain during wakefulness and sleep. Psychology and Behavioral Science International Journal. 2017;2(2):1-7.

10. Ben-Tal A. Computational models for the study of heart-lung interactions in mammals. Wiley Interdiscip Rev Syst Biol Med. 2012;4(2):163-170.

11. Harrington J, Schramm PJ, Davies CR, et al. An electrocardiogrambased analysis evaluating sleep quality in patients with obstructive sleep apnea. Sleep Breath. 2013;17(3):1071-1078.

12. Skotselyas Yu G, Yumatov EA. Correlation relationships between vegetative parameters under experimental emotional stress. Zh Vyssh Nerv Deiat Im I P Pavlova. 1983;33(1):1128-1134.

13. Sudakov KV, Yumatov EA, Tarakanov OP. Cross-correlation vegetative criterion of emotional stress. Journal of Human Physiology. 1995;23(3):87-95.

14. Arakcheev AG, Sivachev AV. Electrocardiologic technique for the study of the functional state of the heart. 2002. $128 \mathrm{p}$.

15. Frolov AV, Frolov GV. Multimedia for Windows. 1996. 284 p.

16. Potapova OV, Potapov VYu, Yumatov EA. Information software for electroencephalographic analysis of mental activity of the brain. Journal of Neurocomputers: development and application. 2016;1:45-50.

17. Yumatov EA, Potapova OV, Potapov VY, et al. The experimental behavioral model and software to identify of mental activity of the brain. Psychology and Behavioral Science International Journal. 2017;2(1):14. 